\title{
Salud Pública, Gestión Sanitaria, Auditoría Clínica y Ciencias Forenses en el Posgrado y Especialización en Estomatología
}

Public Health, Health Management, Clinical Audit and Forensic Sciences in Dentistry as Specialty Postgraduate Training Programs

Jorge Enrique Manrique Chávez ${ }^{1,2, a, b, c, d, e ~}$

\section{RESUMEN}

Objetivo: Sistematizar la experiencia de diseño curricular y gestión de competencias para la creación de programas de posgrado y especialización en Salud Pública, Gestión Sanitaria, Auditoría Clínica y Ciencias Forenses en Estomatología. Material y métodos: Se realizó una revisión histórica y análisis documental del periodo 20112021 de los cursos de educación continua, diplomados y segundas especialidades profesionales desarrollados por el Departamento Académico de Odontología Social en los campos de Salud Pública, Gestión Sanitaria, Auditoría Clínica y Ciencias Forenses en la Unidad de Posgrado y Especialización de la Facultad de Estomatología de la Universidad Peruana Cayetano Heredia. Resultados: Se diseñó un "Modelo de Campos del Conocimiento para el Diseño Curricular y Gestión de Competencias para Programas de Posgrado y Especialización en Salud Pública, Gestión Sanitaria, Auditoría Clínica y Ciencias Forenses en Estomatología" que reúne un conjunto de competencias, ejes específicos (esenciales) y ejes generales (complementarios). Conclusión: A través de un enfoque de Aprendizaje Basado en Competencias y siguiendo las orientaciones de la Educación en Odontología, se estructuraron campos del conocimiento que permitieron la creación de las segundas especialidades profesionales en Salud Pública Estomatológica, Administración y Gestión en Estomatología, Auditoría Odontológica y Odontología Forense en la Facultad de Estomatología de la Universidad Peruana Cayetano Heredia.

PALABRAS CLAVE: Educación en odontología, salud pública, odontología comunitaria, administración de la práctica odontológica, odontología forense, auditoría odontológica.

1 Facultad de Estomatología Roberto Beltrán, Universidad Peruana Cayetano Heredia. Lima, Perú.

2 Instituto de Salud Oral (ISO). Lima, Perú.

a Docente de la Sección Académica de Salud Pública y Gestión de Servicios Odontológicos del Departamento Académico de Odontología Social (DAOS).

b Creador y artífice de los programas de posgrado y especialización en Salud Pública Estomatológica, Administración y Gestión en Estomatología, Odontología Forense y Auditoría Odontológica de la Unidad de Posgrado y Especialización en Estomatología (UPCH).

c Ex-Director Universitario de la Dirección Universitaria de Planificación y Desarrollo de la Universidad Peruana Cayetano Heredia (DUPDEUPCH). Ex-Director Administrativo de la Facultad de Estomatología (FAEST-UPCH). Ex-Director Clínico de la Clínica Dental Docente (CDDFAEST).

d Posgrado en Auditoría Odontológica (ACADAO-Argentina), Odontología Legal y Forense (UIC-España), Evaluación Económica en Salud (UdeA-Colombia), Medicina Basada en Evidencia (UC-Chile) y Compliance Odontológico (FACOP-Brasil) e ORCID ID: https://orcid.org/0000-0002-5119-5405 


\section{ABSTRACT}

Objective: To systematize the experience of curricular design and competencies management for the creation of postgraduate and specialization programs in Public Health, Health Management, Clinical Audit and Forensic Sciences in Stomatology. Material and Methods: A historical review and documentary analysis of the period 2011-2021 of the continuing education courses, diplomas and second professional specialties developed by the Academic Department of Social Dentistry in the fields of Public Health, Health Management, Clinical Audit and Forensic Sciences in the Postgraduate and Specialization Unit of the Faculty of Stomatology of the Universidad Peruana Cayetano Heredia. Results: A "Model of Fields of Knowledge for Curriculum Design and Competency Management for Postgraduate and Specialization Programs in Public Health, Health Management, Clinical Audit and Forensic Sciences in Dentistry" was designed that brings together a set of competencies, specific axes (essential) and general axes (complementary). Conclusions: Through a Competency-Based Learning approach and following the guidelines of Education in Dentistry, fields of knowledge were structured that allowed the creation of second professional specialties in Dental Public Health, Dental Practice Management, Dental Audit and Forensic Dentistry at the Faculty of Stomatology of the Universidad Peruana Cayetano Heredia.

KEYWORDS: Dental Education, public health, community dentistry, dental practice management, forensic dentistry, dental audit.

\section{INTRODUCCIÓN}

En el 2011, el Dr. Roberto Beltrán Neira, profesor fundador y emérito de la Facultad de Estomatología, publicó un artículo en el cual planteó una autocrítica del desarrollo profesional de la Odontología en el Perú, indicando que "Llamamos Deuda Social de la Odontología, al hecho evidente de que la profesión conoce la problemática de salud bucal, tiene los instrumentos, materiales y métodos para solucionarla y no lo ha hecho. Podríamos compararla con un profesional que tiene la droga para curar a una persona enferma y no se la administra. El Odontólogo tiene la obligación moral de mantener la salud bucal de la comunidad, actuando como clínico, educador, investigador y político" (1).

En tal sentido, a modo de respuesta frente a dicha Deuda Social, estableció una visión para saldarla a través de un conjunto de puntos clave que surgen como retos en la formación y desarrollo profesional del Cirujano Dentista del siglo XXI. Estos puntos incluyen la necesidad de reconocer los determinantes sociales con enfoque epidemiológico, reorientar los servicios hacia la promoción de la salud y prevención de las enfermedades durante todo el curso de vida, investigar e innovar en propuestas de desarrollo académico y/o educativo a nivel superior, incorporar la formación y adquisición de competencias en el campo político, desarrollar habilidades para liderar y gerenciar el cambio, impulsar la acción de la profesión conectada con la realidad de la sociedad y del país, vigilar, monitorizar y actuar sobre el cambio como variable constante en el desarrollo de la profesión, evaluar los resultados e impactos de las intervenciones y formular propuestas de desarrollo científico, académico y social con innovación (1).

Es así como, a partir del 2011, desde la Sección Académica de Salud Pública y Gestión de Servicios Odontológicos del Departamento Académico de Odontología Social (DAOS) de la Facultad de Estomatología, se inició un proceso de diversificación de la oferta académica en la Unidad de Posgrado y Especialización con propuestas con "Fuerza Innovadora" (2), centradas en cuatro ejes esenciales para el desarrollo de la profesión estomatológica en los campos de la Salud Pública, Gestión Sanitaria, Auditoría Clínica y Ciencias Forenses con excelencia académica, relevancia científica y compromiso social (figura 1):

1. Construcción de políticas públicas y acciones sociales en materia de salud bucal.

2. Liderazgo en la gestión integral de servicios odontológicos en el sistema de salud.

3. Garantía de la calidad asistencial y seguridad de las prestaciones estomatológicas.

4. Responsabilidad profesional frente a las implicancias odontológico-legales y sociales. 


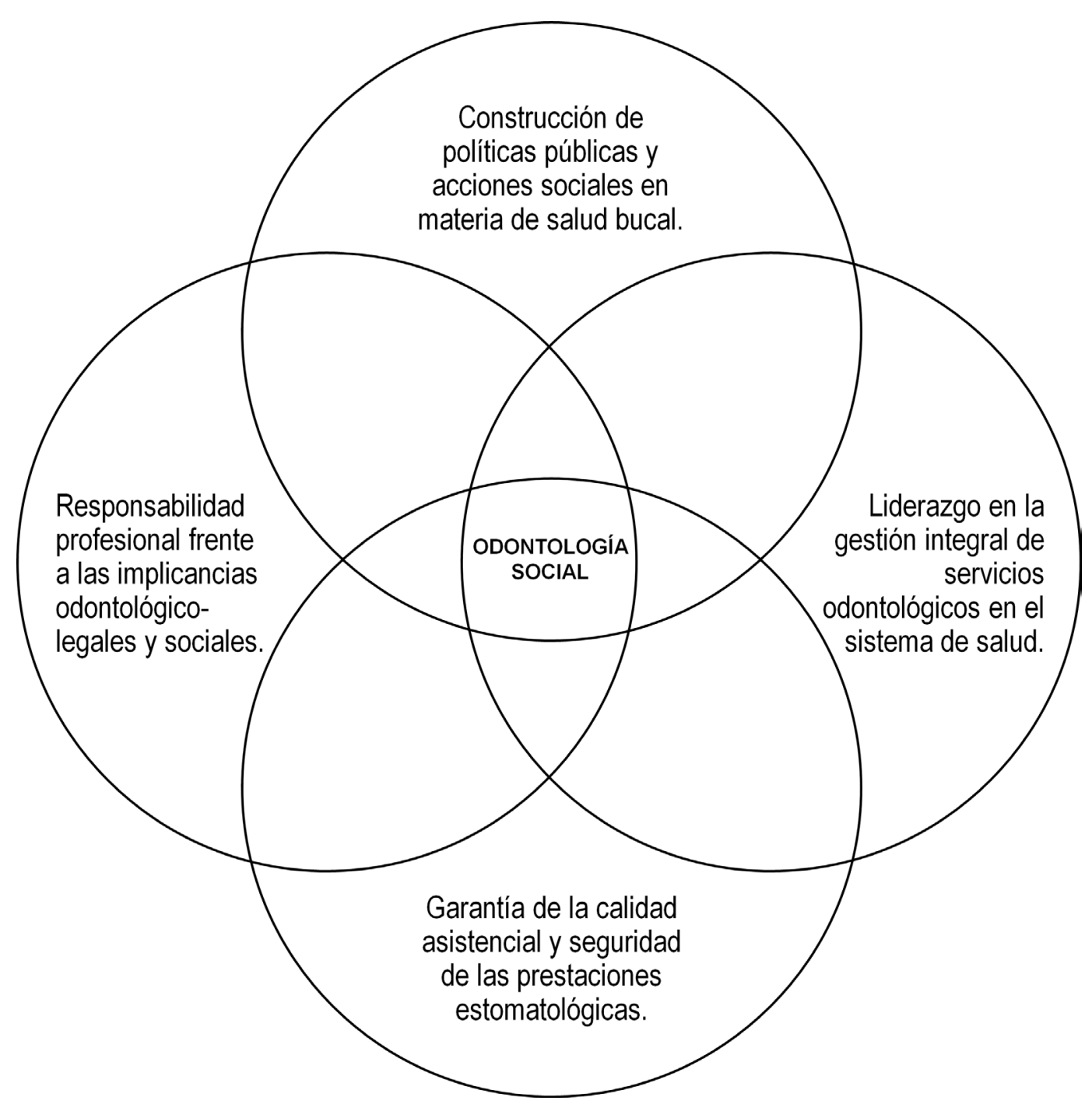

Figura 1. Ejes esenciales para el diseño curricular y gestión de competencias para programas de posgrado y especialización en Salud Pública, Gestión Sanitaria, Auditoría Clínica y Ciencias Forenses en Estomatología. (Jorge E. Manrique-Chávez. Facultad de Estomatología-UPCH. 2011)

\section{PROCESO DE DIVERSIFICACIÓN CURSOS Y DIPLOMADOS}

E1 26 de noviembre de 2011 se realizó por primera vez el curso de educación continua en "Auditoría Odontológica y Odontología Forense", el cual fue seguido por el también primer curso sobre "Salud Pública y Gestión Odontológica" el 3 de diciembre del mismo año, ambos organizados desde la Unidad de Posgrado y Especialización de la Facultad de Estomatología. El impacto de ambos cursos dio pase a la organización de dos nuevos: el primero, "Gerencia Odontológica y Salud Pública Bucal"; el segundo, "Odontología Legal, Forense y Auditoría Odontológica", celebrados el 18 y 19 de abril de 2012, respectivamente.
En ese mismo año, a solicitud del Consejo de Facultad de fecha 17 de octubre de 2011, se aprobaron cuatro programas de Diplomados para ser desarrollados durante el periodo mayo de 2012 abril de 2013, siendo estos los primeros en centrarse exclusivamente en áreas de Salud Pública, Gestión Sanitaria, Auditoría Clínica y Ciencias Forenses en Estomatología (tabla 1).

Posteriormente, nuevamente a solicitud del Consejo de Facultad de fecha 12 de noviembre de 2012, se aprobaron cuatro nuevos programas de Diplomados para el periodo correspondiente a mayo de 2013 - abril de 2014, ampliando así su catálogo académico y complementando lo antes trabajado 
Tabla 1. Programas Académicos de Diplomados en áreas de Salud Pública, Gestión Sanitaria, Auditoría Clínica y Ciencias Forenses en Estomatología 2011-2013. (Jorge E. Manrique-Chávez. Facultad de Estomatología-UPCH. 2011)

\begin{tabular}{cc}
\hline Diplomado & Resolución Rectoral \\
\hline $\begin{array}{c}\text { Gestión Odontológica y Gerencia de Servicios } \\
\text { Estomatológicos }\end{array}$ & $\mathrm{N}^{\mathrm{o}} 1088-2011-\mathrm{UPCH}-\mathrm{CU}$ \\
$\begin{array}{c}\text { Auditoría Odontológica y Garantía de la Calidad } \\
\text { Estomatológica }\end{array}$ & $\mathrm{N}^{\mathrm{o}}$ 1089-2011-UPCH-CU \\
$\begin{array}{c}\text { Salud Pública Estomatológica y Odontología } \\
\text { Comunitaria }\end{array}$ & $\mathrm{N}^{\mathrm{o}}$ 1090-2011-UPCH-CU \\
Odontología Legal, Forense y Criminalística & $\mathrm{N}^{\mathrm{o}} 1091-2011-\mathrm{UPCH}-\mathrm{CU}$ \\
\hline
\end{tabular}

Tabla 2. Programas Académicos de Diplomados en áreas de Salud Pública, Gestión Sanitaria, Auditoría Clínica y Ciencias Forenses en Estomatología 2012-2014. (Jorge E. Manrique-Chávez. Facultad de Estomatología-UPCH. 2012)

\begin{tabular}{cc}
\hline Diplomado & Resolución Rectoral \\
\hline $\begin{array}{c}\text { Dirección Odontológica y } \\
\text { Administración de Empresas Estomatológicas }\end{array}$ & $\mathrm{N}^{\mathrm{o}}$ 093-2013-UPCH-CU \\
$\begin{array}{c}\text { Peritaje en Odontología y Valoración del Daño } \\
\text { Estomatológico }\end{array}$ & $\mathrm{N}^{\mathrm{o}}$ 094-2013-UPCH-CU \\
$\begin{array}{c}\text { Salud Bucal Colectiva y Odontología Familiar } \\
\text { Seguridad del Paciente Odontológico y Gestión dela } \\
\text { Calidad Estomatológica }\end{array}$ & $\mathrm{N}^{\mathrm{o}}$ 095-2013-UPCH-CU \\
& $\mathrm{N}^{\mathrm{o}}$ 096-2013-UPCH-CU \\
\hline
\end{tabular}

con nuevas herramientas, técnicas, métodos y procedimientos de aplicación en el lado "no clínico" de la profesión odontológica (tabla 2).

Durante el periodo 2012-2014 se desarrollaron también un conjunto de cursos que formaron parte del programa de Capacitación Estomatológica Integral (CEI): "Fundamentos en Auditoría Odontológica y Odontología Forense", "Fundamentos en Salud Pública Estomatológica y Gestión Odontológica", "Fundamentos en Gerencia y Sistemas de Atención en Estomatología" y "Fundamentos en Legislación y Seguridad del Paciente en Estomatología”. Además, con base a la experiencia adquirida, se impulsaron cuatro ejes complementarios para el desarrollo de la profesión estomatológica en la Salud Pública, Gestión Sanitaria, Auditoría Clínica y Ciencias Forenses, con enfoque creativo y diversificador en propuestas innovadoras que permitan afianzar la docencia en nuevos espacios de especialización y consolidar nuevas líneas de investigación (figura 2):

1. Abordaje integral por curso de vida con servicios reorientados a la promoción y prevención.
2. Innovación y emprendimiento estratégico de modelos de atención sanitaria y planes de acción.

3. Implementación de sistemas de inteligencia, vigilancia, prevención y control de riesgos.

4. Reconocimiento y medición de inequidades, gradiente social y determinante epidemiológica.

\section{CREACIÓN DE SEGUNDASESPECIALIDADES PROFESIONALES}

Durante el 2013, en un proceso natural de evolución, habiendo impulsado un grupo notable de cursos de educación continua y desarrollado hasta ocho programas de Diplomados, el siguiente escalón fue llegar a la cúspide de la formación de competencias de especialización para el abordaje de casuística de mediana y alta complejidad en las áreas de Salud Pública, Gestión Sanitaria, Auditoría Clínica y Ciencias Forenses en Odontología. En tal sentido, a solicitud del Consejo de Facultad con fecha 14 de octubre de 2013, se aprobó la creación de cuatro programas de Segundas Especialidades Profesionales: Salud Pública Estomatológica, Administración y Gestión en Estomatología, Auditoría Odontológica y Odontología Forense (tabla 3). 


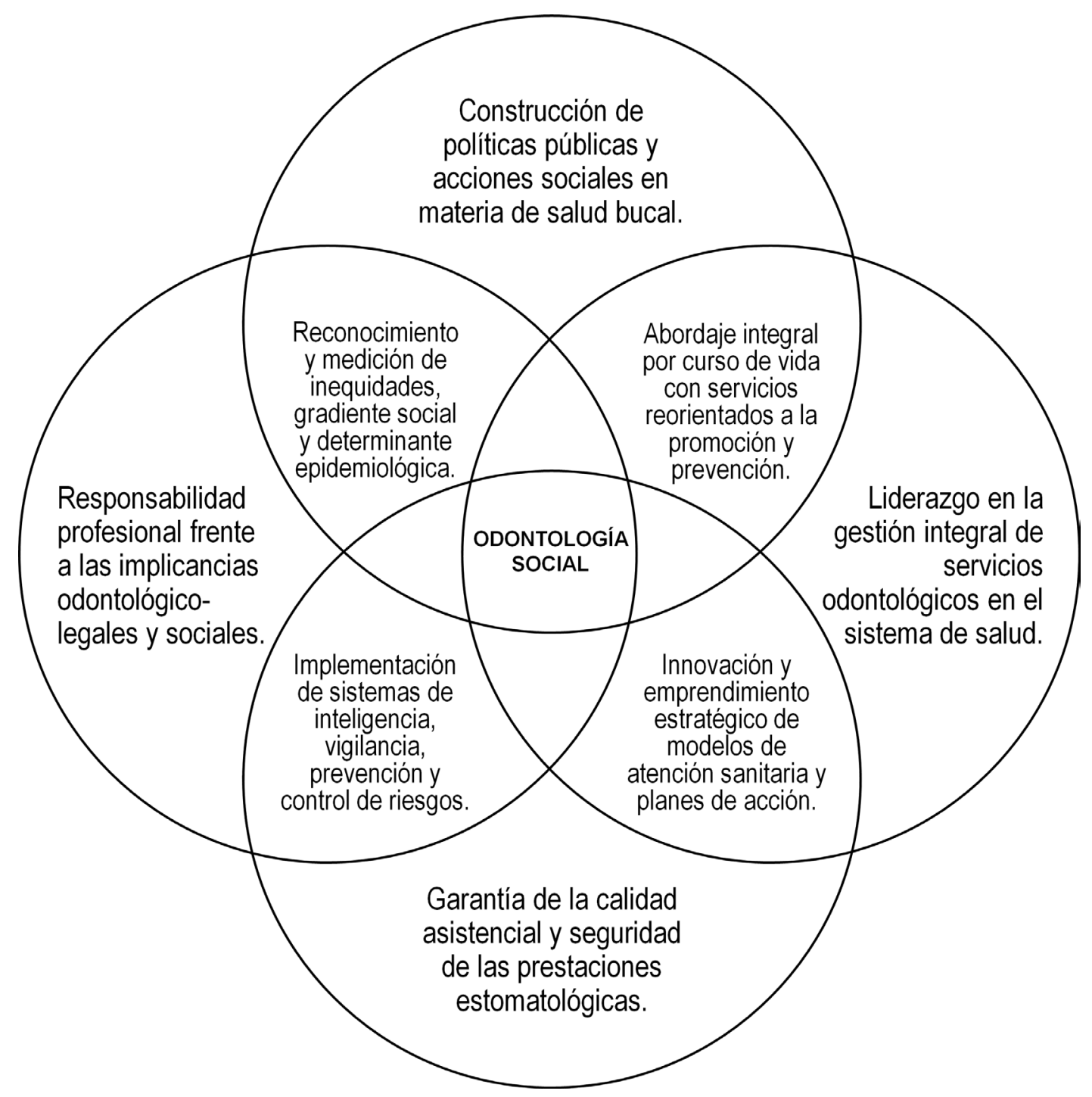

Figura 2. Ejes complementarios para el diseño curricular y gestión de competencias para programas de posgrado y especialización en Salud Pública, Gestión Sanitaria, Auditoría Clínica y Ciencias Forenses en Estomatología. (Jorge E. Manrique-Chávez. Facultad de Estomatología-UPCH. 2011)

Tabla 3. Programas Académicos de Segundas Especialidades Profesionales en áreas de Salud Pública, Gestión Sanitaria, Auditoría Clínica y Ciencias Forenses en Estomatología 2013-2015. (Jorge E. Manrique-Chávez. Facultad de EstomatologíaUPCH. 2013)

\begin{tabular}{|c|c|}
\hline Segunda Especialidad Profesional & Resolución Rectoral \\
\hline Salud Pública Estomatológica & $\mathrm{N}^{\mathrm{o}} 1121-2013-\mathrm{UPCH}-\mathrm{CU}$ \\
\hline Administración y Gestión en Estomatología & $\mathrm{N}^{\circ} 1122-2013-\mathrm{UPCH}-\mathrm{CU}$ \\
\hline Auditoría Odontológica & $\mathrm{N}^{\mathrm{o}} 1123-2013-\mathrm{UPCH}-\mathrm{CU}$ \\
\hline Odontología Forense & $\mathrm{N}^{\circ} 1124-2013-\mathrm{UPCH}-\mathrm{CU}$ \\
\hline
\end{tabular}


A finales del 2014, se sumarían nuevos cursos sobre "Principios de Gestión del Riesgo en Estomatología", "Principios de Peritaje y Auditoría en Estomatología", "Principios de Políticas y Normativas en Estomatología" y "Principios de Salud Ocupacional en Estomatología" y ya para el 2015 se abordarían otras áreas temáticas como "Seguros y Aseguramiento en Estomatología", "Modelos de Negocio y Franquicias Odontológicas", "Auditoría Odontológica y Odontología Forense", "Salud Pública Estomatológica y Gestión Odontológica", "Peritaje y Auditoría en Estomatología", "Salud Ocupacional en Estomatología", "Gestión de la Calidad y Atención al Cliente en Estomatología" y "Certificación ISO y Auditorías de Calidad en Estomatología".

Para finales del 2015 se graduaría la primera promoción de especialistas en modalidad escolarizada en "Salud Pública Estomatológica", en "Administración y Gestión en Estomatología", en "Auditoría Odontológica" y en "Odontología Forense" de la Facultad de Estomatología de la
Universidad Peruana Cayetano Heredia (3). Esto no sólo sería un hito académico institucional a nivel de posgrado, sino que daría paso a una nueva etapa de formación con el inicio del Residentado Odontológico que incorporaría el programa de "Salud Pública Estomatológica" en el marco del Decreto Supremo $\mathrm{N}^{\circ}$ 009-2013-SA, así como el reconocimiento de las especialidades de "Administración y Gestión en Estomatología" y de "Auditoría Odontológica" en el cuadro de especialidades consensuadas con el Colegio Odontológico del Perú y reconocidas por Decreto Supremo $\mathrm{N}^{\circ}$ 032-2016-SA, siendo la Facultad de Estomatología de la Universidad Peruana Cayetano Heredia, la única en el Perú en brindar dichos programas en su portafolio académico de posgrado.

\section{CONSTRUCCIÓN Y DESARROLLO DE CAMPOS DE ACCIÓN}

Con la creación de las nuevas especialidades se cubre con el total de competencias requeridas a nivel de posgrado y especialización para atender las

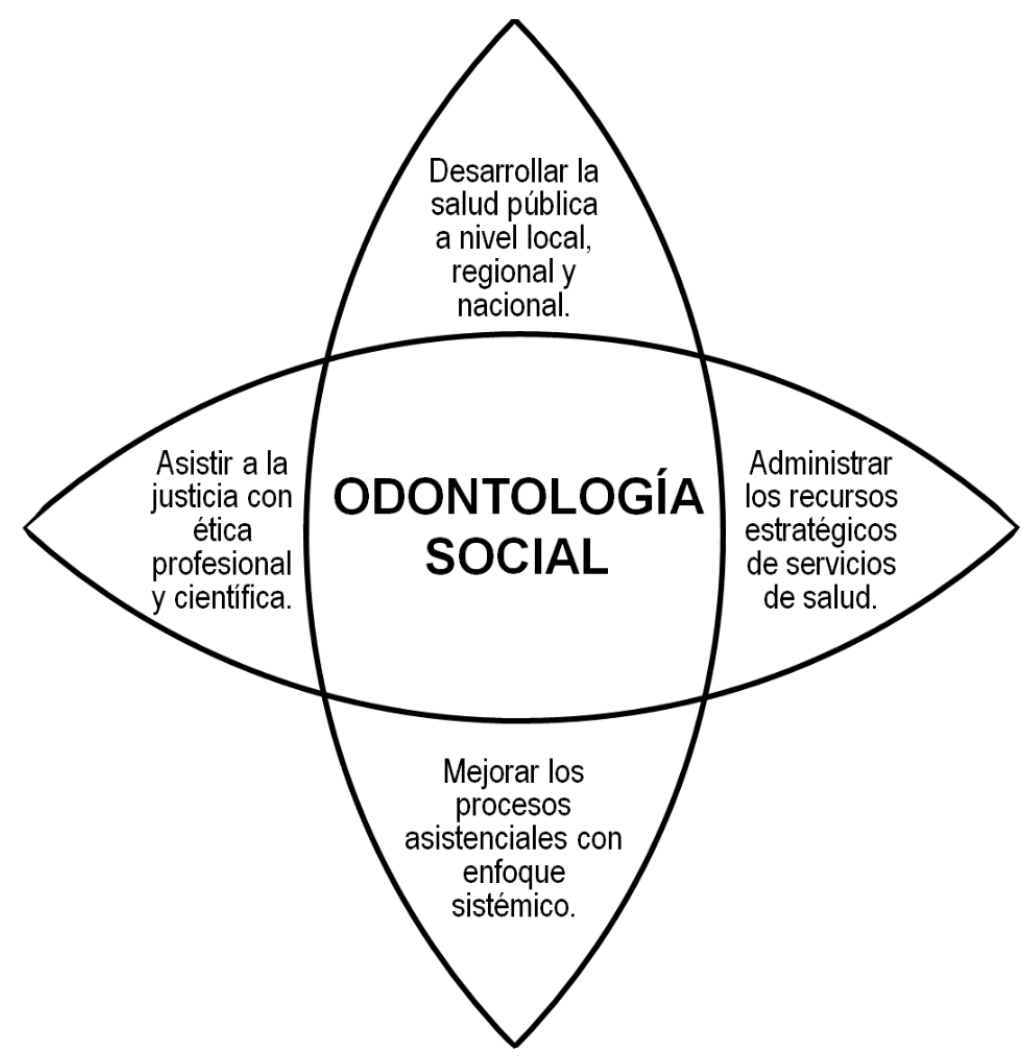

Figura 3. Competencias requeridas por los programas de segunda especialización profesional orientados a los campos de Salud Pública, Gestión Sanitaria, Auditoría Clínica y Ciencias Forenses en Estomatología. (Jorge E. Manrique-Chávez. Facultad de Estomatología-UPCH. 2011) 
necesidades de salud, yendo más allá de las necesidades estomatológicas, médicas y/o quirúrgicas del sistema estomatognático o del individuo, para cubrir con los problemas sociales que aquejan a las familias y comunidades en sus entornos socioeconómicos, socioculturales y sociodemográficos, acorde a la clasificación ordenada de competencias en el campo de la Educación Odontológica de forma jerarquizada y sistemática (4), donde se desarrolla el Cirujano Dentista como artífice de la expansión y consolidación de su propio campo profesional, desarrollando espacios de formación orientados al bien común entre los diversos sectores en el que se desempeña (5) y en respuesta a la constante exploración de un espacio virtuoso de crecimiento profesional e innovación académica (6) (figura 3):

1. Desarrollar la salud pública a nivel local, regional y nacional.

2. Administrar los recursos estratégicos de servicios de salud.
3. Mejorar los procesos asistenciales con enfoque sistémico.

4. Asistir a la justicia con ética profesional y científica.

Para el 2016 se desarrollaron nuevos cursos de educación continua sobre "Odontología Social y Salud Pública I y II", "Gestión Odontológica y Administración en Salud I y II", "Odontología Legal y Ciencias Forenses I y II” y “Auditoría Odontológica y Supervisión Asistencial I y II" que fueron base conceptual para la creación del "Diplomado en Gestión y Desarrollo Odontológico" que se extendió hasta el 2017. Este diplomado se centró en el desarrollo de competencias de profesionales en cuatro campos de la Odontología Social, integrando en un solo plan curricular aspectos de Salud Pública Estomatológica, Auditoría Odontológica, Administración y Gestión en Estomatología y Odontología Forense. Planteó una formación integral basada en competencias, por lo que su estructura curricular permitía adquirir

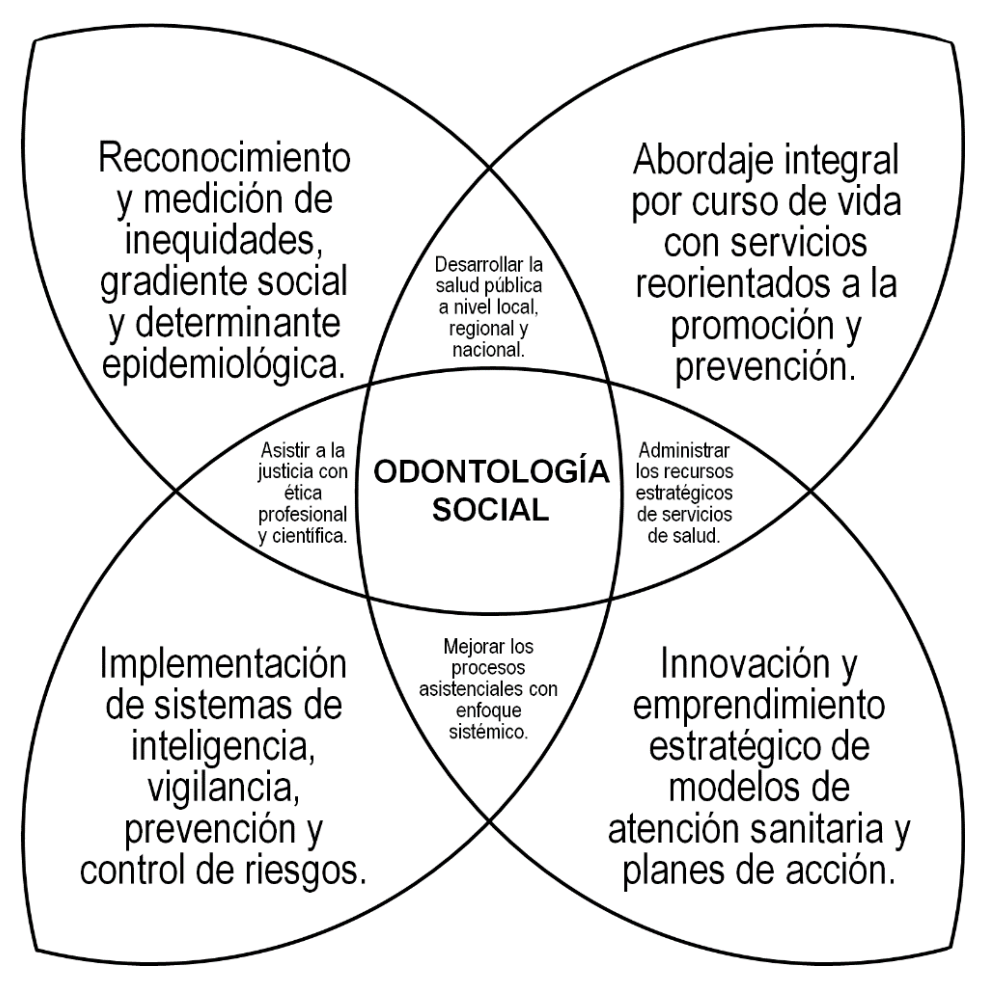

Figura 4. Campo de acción de la Odontología Social a nivel de programas de posgrado y especialización en las áreas profesionales de Salud Pública, Gestión Sanitaria, Auditoría Clínica y Ciencias Forenses en Estomatología. (Jorge E. Manrique-Chávez. Facultad de Estomatología-UPCH. 2011) 
progresivamente herramientas conceptuales, metodológicas y operativas para su aplicación en la práctica odontológica en establecimientos de salud a nivel público y/o privado.

La estructura curricular del "Diplomado en Gestión y Desarrollo Odontológico" incluyó 18 cursos distribuidos en 9 módulos que integraron las cuatro competencias requeridas por los programas de segunda especialización profesional orientados a los campos de Salud Pública, Gestión Sanitaria, Auditoría Clínica y Ciencias Forenses en Estomatología con los cuatro ejes complementarios, de modo tal que dispuso una visión holística de los problemas sociales que afectan al individuo, familia y comunidad, incluyendo al propio Cirujano Dentista como actor clave del sistema de salud en los subsectores del campo público (gubernamental) y del privado (particular), adhiriéndose a los campos de acción del Departamento Académico de Odontología Social (figura 4).

\section{ESTRUCTURACIÓN Y DESPLIEGUE DE UN MODELO}

El 2017 trajo consigo la mayor variedad de cursos de "Gerencia y Marketing en Salud", "Salud Pública y Gestión en Salud", "Auditoría y Supervisión en Salud", "Peritaje y Derechos en Salud", "Administración de Negocios en Salud", "Gerencia Social en Salud", "Finanzas, Economía, Contabilidad \& Tributación en Odontología", "Odontología Legal, Forense y Criminalística", "Odontología Ocupacional y Seguridad en el Trabajo", "Salud Familiar y Comunitaria en Odontología", "Salud Ocupacional y Odontología del Trabajo", "Auditoría Odontológica: Auditoría en Salud \& Auditoría de Gestión Clínica", "Evaluación, Control y Supervisión de Consultorios y Centros Odontológicos", "Gestión Odontológica: Dirección Médica \& Gestión Clínica del Consultorio", "Auditoría Clínica y Peritaje Judicial en Odontología", "Odontología Familiar y Salud Comunitaria en Odontología", "Salud Pública

Tabla 4. Estructuración de Campos del Conocimiento para el Diseño Curricular y Gestión de Competencias para Programas de Posgrado y Especialización en Salud Pública, Gestión Sanitaria, Auditoría Clínica y Ciencias Forenses en Estomatología. (Jorge E. Manrique-Chávez. Facultad de Estomatología-UPCH. 2011)

\begin{tabular}{|c|c|c|c|c|}
\hline $\begin{array}{c}\text { Especialización } \\
\text { Profesional (General) }\end{array}$ & $\begin{array}{c}\text { Campo de } \\
\text { Competencias } \\
\text { Profesionales }\end{array}$ & $\begin{array}{l}\text { Ejes Esenciales } \\
\text { Especializados }\end{array}$ & $\begin{array}{c}\text { Ejes Generales } \\
\text { Complementarios }\end{array}$ & $\begin{array}{l}\text { Especialización } \\
\text { Profesional } \\
\text { (Específico) }\end{array}$ \\
\hline $\begin{array}{l}\text { Salud Pública } \\
\text { Estomatológica }\end{array}$ & $\begin{array}{l}\text { Desarrollar la salud } \\
\text { pública a nivel local, } \\
\text { regional y nacional. }\end{array}$ & $\begin{array}{l}\text { Construcción de } \\
\text { políticas públicas } \\
\text { y acciones sociales } \\
\text { en materia de salud } \\
\text { bucal. }\end{array}$ & $\begin{array}{l}\text { Abordaje integral por } \\
\text { curso de vida con } \\
\text { servicios reorientados } \\
\text { a la promoción y } \\
\text { prevención. }\end{array}$ & $\begin{array}{l}\text { Salud Familiar y } \\
\text { Comunitaria en } \\
\text { Odontología }\end{array}$ \\
\hline $\begin{array}{l}\text { Administración y } \\
\text { Gestión en } \\
\text { Estomatología }\end{array}$ & $\begin{array}{l}\text { Administrar los recursos } \\
\text { estratégicos de los } \\
\text { servicios de salud. }\end{array}$ & $\begin{array}{l}\text { Liderazgo en la } \\
\text { gestión integral } \\
\text { de servicios } \\
\text { odontológicos en el } \\
\text { sistema de salud. }\end{array}$ & $\begin{array}{c}\text { Innovación y } \\
\text { emprendimiento } \\
\text { estratégico de } \\
\text { modelos de atención } \\
\text { sanitaria y planes de } \\
\text { acción. }\end{array}$ & $\begin{array}{c}\text { Odontología } \\
\text { Empresarial y } \\
\text { Marketing Dental }\end{array}$ \\
\hline Auditoría Odontológica & $\begin{array}{c}\text { Mejorar los procesos } \\
\text { asistenciales con } \\
\text { enfoque sistémico. }\end{array}$ & $\begin{array}{l}\text { Garantía de la } \\
\text { calidad asistencial } \\
\text { y seguridad de } \\
\text { las prestaciones } \\
\text { estomatológicas. }\end{array}$ & $\begin{array}{l}\text { Implementación } \\
\text { de sistemas de } \\
\text { inteligencia, } \\
\text { vigilancia, prevención } \\
\text { y control de riesgos. }\end{array}$ & $\begin{array}{l}\text { Odontología } \\
\text { Ocupacional }\end{array}$ \\
\hline Odontología Forense & $\begin{array}{c}\text { Asistir a la justicia } \\
\text { con ética profesional y } \\
\text { científica. }\end{array}$ & $\begin{array}{c}\text { Responsabilidad } \\
\text { profesional frente } \\
\text { a las implicancias } \\
\text { odontológico-legales } \\
\text { y sociales. }\end{array}$ & $\begin{array}{c}\text { Reconocimiento } \\
\text { y medición de } \\
\text { inequidades, gradiente } \\
\text { social y determinante } \\
\text { epidemiológica. }\end{array}$ & $\begin{array}{l}\text { Epidemiología } \\
\text { Estomatológica }\end{array}$ \\
\hline
\end{tabular}




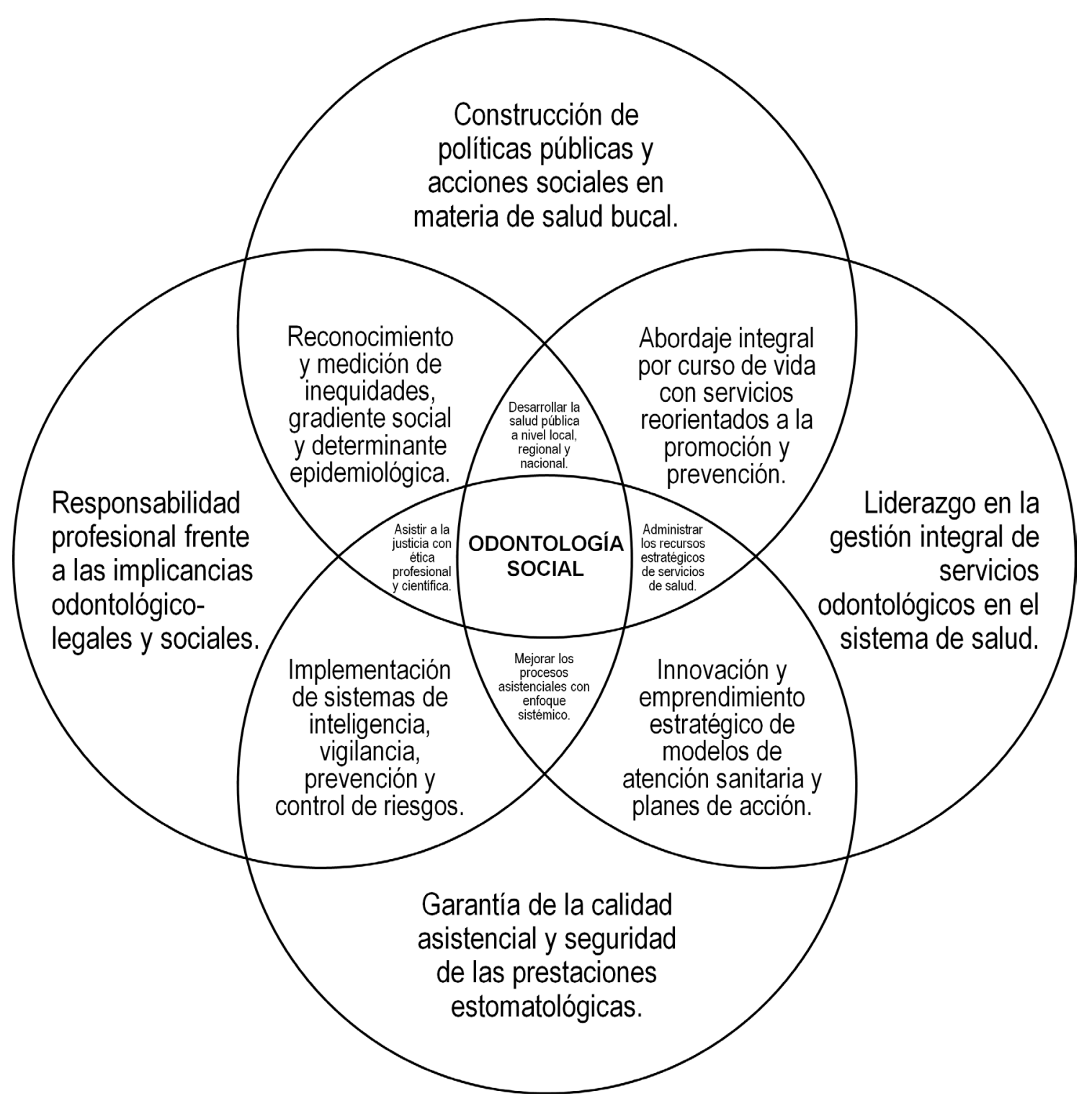

Figura 5. Conjunto de competencias, ejes específicos (esenciales) y ejes generales (complementarios) que definen los diversos campos de especialización en Salud Pública, Gestión Sanitaria, Auditoría Clínica y Ciencias Forenses en Estomatología. (Jorge E. Manrique-Chávez. Facultad de Estomatología-UPCH. 2011)

Dental: Investigación en Salud \& Epidemiología”, "Odontología Legal: Aspectos Legales \& Procesos Judiciales", "Odontología Forense: Ciencias Forenses, Periciales \& Criminalística", "Administración y Gestión Empresarial en Odontología", "Gerencia y Marketing Estratégico en Odontología" y "Finanzas y Contabilidad para Empresas de Salud", reafirmando así el posicionamiento del Cirujano Dentista en las áreas de Salud Pública, Gestión Sanitaria, Auditoría Clínica y Ciencias Forenses.
Posteriormente, durante el periodo 2018-2020, se continuaron desarrollando nuevas ediciones de los Diplomados y Segundas Especialidades Profesionales, intercalando áreas temáticas, junto a un amplio catálogo de cursos de posgrado en "Contabilidad en Odontología: Obligaciones Tributarias del Cirujano Dentista", "Odontología Ocupacional: Seguridad y Salud en el Trabajo (SST)", "Salud Pública Estomatológica: Gobierno, Políticas y Gestión Pública en Salud”, “Odontología Forense: Biometría y Patrones para la Identificación 
Humana", "Gerencia y Marketing en Odontología: Estrategias Empresariales para la Clínica Dental”, "Epidemiología Oral: Índices y Encuestas de Salud Bucodental", "Odontología Legal: Gestión de Quejas, Reclamos y Denuncias", "Auditoría Odontológica: Evaluación, Control y Supervisión de IPRESS", "Administración y Gestión en Estomatología: Dirección del Centro Odontológico", "Salud Familiar y Comunitaria en Odontología: Atención por Etapas y Curso de Vida", "Negocios y Emprendimiento en Odontología: Modelos de Negocios, Redes y Franquicias en Salud", "Supervisión Asistencial en Odontología: Registro, Categorización y Gestión de Riesgos en Salud”, “Auditoría Odontológica \& Peritaje Odontológico" y "Odontología Ocupacional \& Seguridad en el Trabajo".

Finalmente, para el 2021, cursos de posgrado en "Finanzas y Contabilidad para Servicios Odontológicos", "Odontología Ocupacional y Seguridad en el Trabajo", "Gerencia y Marketing para Servicios Odontológicos", "Dirección y Gestión para Servicios Odontológicos", "Salud Pública y Epidemiología en Odontología", "Salud Familiar y Comunitaria en Odontología”, "Auditoría Odontológica y Calidad Odontológica", "Peritaje

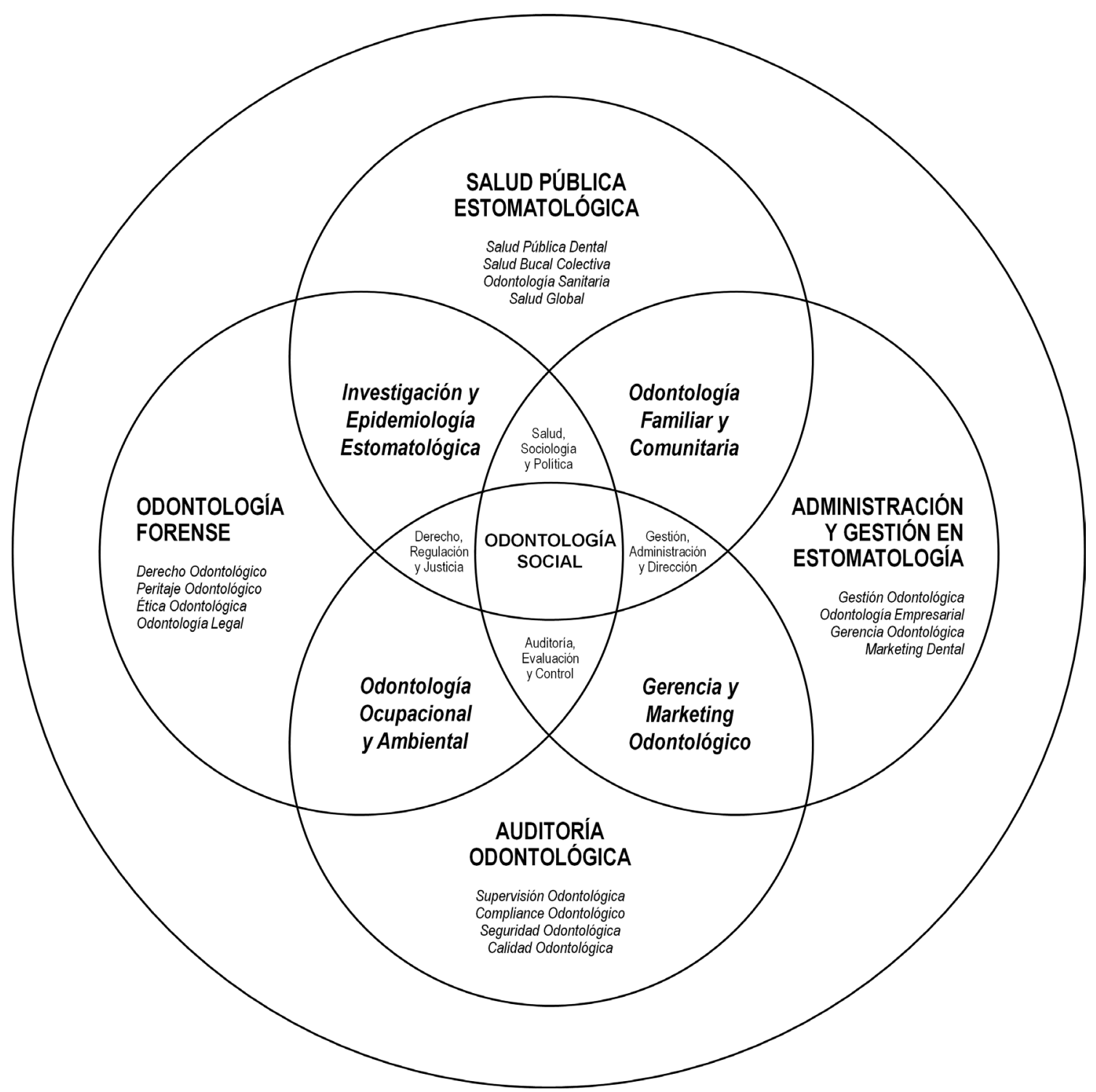

Figura 6. Modelo de Campos del Conocimiento para el Diseño Curricular y Gestión de Competencias para Programas de Posgrado y Especialización en Salud Pública, Gestión Sanitaria, Auditoría Clínica y Ciencias Forenses en Estomatología. (Jorge E. Manrique-Chávez. Facultad de Estomatología-UPCH. 2011) 
Odontológico y Odontología Legal”, “Odontología Forense y Ciencias Forenses" y "Teleodontología y Salud Global en Odontología" se sumaron al amplio catálogo de educación continua del Departamento Académico de Odontología Social (DAOS) de la Facultad de Estomatología, con el compromiso continuar con el legado de nuestros fundadores en la búsqueda y elaboración de programas con alto valor académico, con excelencia, compromiso social e innovación, cubriendo con un conjunto de competencias, ejes específicos (esenciales) y ejes generales (complementarios) que definen los diversos campos de especialización en Salud Pública, Gestión Sanitaria, Auditoría Clínica y Ciencias Forenses en Estomatología (tabla 4).

En tal sentido, diez años después de haber iniciado este proceso de diversificación y en pleno proceso de transición epidemiológica y demográfica por la pandemia de COVID-19 y su inevitable repercusión nacional e internacional en aspectos políticos, sociales y económicos, el desarrollo de la profesión estomatológica en la Salud Pública, en la Gestión Sanitaria, en la Auditoría Clínica y en las Ciencias Forenses se hace más necesaria que nunca. Por tanto, la Sección Académica de Salud Pública y Gestión de Servicios Odontológicos del Departamento Académico de Odontología Social (DAOS) de la Facultad de Estomatología, continúa sumando esfuerzos para consolidar sus programas de "Salud Pública Estomatológica" (7), y de "Administración y Gestión en Estomatología" (8).

Además, sumado a todo lo antes desarrollado, emergen otros espacios para el desarrollo de la Investigación Estomatológica, Odontología Ocupacional, Epidemiología Oral, Salud Global, Derecho Odontológico, Odontología Sostenible, Marketing Dental, Gerencia Odontológica, Odontología Familiar, Compliance Odontológico, Odontología Empresarial, Odontología Deportiva, Informática Odontológica y Teleodontología, que no son ajenos a la evolución profesional y forman parte del proceso de creación de las Segundas Especialidades Profesionales en "Salud Pública Estomatológica" (9), "Administración y Gestión en Estomatología" (10), “Auditoría Odontológica" (11), y "Odontología Forense" (12), de la Facultad de Estomatología de la Universidad Peruana Cayetano Heredia construyendo un "Modelo de Campos del Conocimiento para el
Diseño Curricular y Gestión de Competencias para Programas de Posgrado y Especialización en Salud Pública, Gestión Sanitaria, Auditoría Clínica y Ciencias Forenses en Estomatología" (figura 6), con enfoque en el Aprendizaje Basado en Competencias (13), y siguiendo las orientaciones de la Educación en Odontología (14).

\section{Correspondencia:}

Jorge Enrique Manrique Chávez

Jirón Pedro Martinto 114, Barranco. Lima-Perú.

Teléfono: (51) 987703140

Correo electrónico: jorge.manrique.ch@upch.pe

\section{REFERENCIAS BIBLIOGRÁFICAS}

1. Beltrán-Neira RJ. Deuda social de la odontología. Rev Estomatol Herediana. 2011; 21(3):175-8.

2. Beltrán-Neira RJ. Fuerza innovadora. Rev Estomatol Herediana. 2015; 25(3):177-8.

3. Mas J. Posgrado: 25 años de excelencia académica. Rev Estomatol Herediana. 2015; 25(4):253-4.

4. Beltrán-Neira RJ, Ikeda MC. Taxonomía de competencias en educación odontológica. Rev Estomatol Herediana. 2004; 14(1-2):107-11.

5. Huanambal V. Universidad-Empresa-Estado: Alineando intereses en función del bien común. Rev Estomatol Herediana. 2017; 27(1):3-4.

6. Huanambal V. La búsqueda del círculo virtuoso de la Investigación, Desarrollo e Innovación. Rev Estomatol Herediana. 2014; 24(3):137-8.

7. Manrique-Chávez JE. Salud pública estomatológica. Rev Estomatol Herediana. 2016; 26(4):203-5.

8. Manrique-Chávez JE. Administración y gestión en estomatología. Rev Estomatol Herediana. 2018; 28(2):69-71

9. Manrique-Chávez JE, Manrique-Guzmán JA, ChávezReátegui BC, Manrique-Chávez CB. Salud Pública Dental: Caja de Herramientas para Estudios Epidemiológicos de Salud Bucal. Lima: Instituto de Salud Oral; 2019.

10. Manrique-Chávez JE, Chávez-Reátegui BC, Manrique-Guzmán JA, Manrique-Chávez CB. Gestión Odontológica: Caja de Herramientas para Modelos y Planes de Negocios Odontológicos. Lima: Instituto de Salud Oral; 2019.

11. Manrique-Chávez JE, Manrique-Guzmán JA, Manrique-Chávez CB, Chávez-Reátegui BC. Auditoría Odontológica: Caja de Herramientas para Auditorías y Control Prestacional Odontológico. Lima: Instituto de Salud Oral; 2019.

12. Manrique-Chávez JE, Manrique-Chávez CB, ChávezReátegui BC, Manrique-Guzmán JA. Odontología 
Forense: Caja de Herramientas para Identificación y Análisis Forense Odontológico. Lima: Instituto de Salud Oral; 2019.

13. Ikeda MC, Beltrán-Neira RJ. Aprendizaje Basado en Competencias. Lima: Universidad Peruana Cayetano Heredia; 2006.
14. Beltrán-Neira RJ. Educación en Odontología - Manual del Profesor. 2da. Edición. Lima: Universidad Peruana Cayetano Heredia; 2019.

Recibido : 08-07-2021

Aceptado : 17-07-2021 